\section{Gerhard Schilling}

Präsident, Verein für Hausarztmedizin im Kanton Schaffhausen
Korrespondenz:

Dr. med. Gerhard Schilling Chlini Schanz 42

CH-8260 Stein am Rhein

gerhard.schilling@hin.ch
Sehr geehrter Herr Manser

Sie lancieren bei uns im Kanton SH und anderswo ein Pseudo-Hausarztmodell («Hausarztversicherung»), das auf keine echte und partnerschaftliche Kooperation zwischen den beteiligten Partnern abstützt. Dabei stellen Sie von unseren 73 Vereinsmitgliedern lediglich deren 28 auf der Hausarztliste zur Auswahl. Trotz mehrfacher Nachfrage zu den Kriterien für diese willkürliche Auswahl sind Sie nicht in der Lage, plausible, transparente und nachvollziehbare Auswahlkriterien zu nennen. Das wichtigste Kriterium scheint die Limite von maximal $40 \%$ zur Mitarbeit zugelassenen Ärzte zu sein.

Mit einstimmig beschlossenem Auftrag und im Namen der Mitglieder des Vereins für Hausarztmedizin im Kanton Schaffhausen protestiere ich aufs schärfste gegen diese willkürliche Geschäftspolitik und fordere Sie umgehend zur Sistierung dieses Produktes auf. Sämtliche Vereinsmitglieder verweigern eine Mitarbeit am Modell der «Hausarzt»versicherung der Helsana! Wir behalten uns öffentliche Aktionen zur Aufklärung der Patienten und Warnung vor Ihrem Geschäftsgebaren vor.

Wir waren einmal Partner. Von 1996 bis Juni 2002 fand eine kooperative Zusammenarbeit zwischen der Helsana und dem Hausarztverein Schaffhausen (HAV-SH) statt, bis Sie in einer Nacht-und-Nebel-Aktion kurzfristig während des laufenden Versicherungsjahres einseitig den Vertrag unter unwahren Angaben (angebliches Nichtrentieren der Hausarztmodelle) aufkündigten und die Patienten zu einer Nachzahlung für das restliche Jahr verpflichteten. In Ihrer neusten Pressemitteilung vom November 2006 strafen Sie aber Ihre eigene, damalige Aussage Lügen, bestätigen Sie doch selber, dass die Hausarztversicherungen (Zitat): «deutlich weniger Kosten verursachen». Die Spatzen pfeifen es von den Dächern: Der damalige Rückzieher erfolgte vermutlich wegen des erlittenen Börsenspekulationsverlustes der angelegten Prämiengelder.

Mit Ihrer eingeschränkten Hausarztwahlliste diskriminieren Sie einen grossen Teil der Bevölkerung. Beispiel Thayngen SH: Von vier Landpraktikern steht nur einer zur Auswahl, 75\% der Versicherten der Helsana sind somit gezwungen, höhere Prämien zu bezahlen, ein Arztwechsel ist aus Gründen der eingeschränkten Kapazität der Landärzte unmöglich und auch unerwünscht.

Mit Ihrer Geschäftspolitik betätigen Sie sich als Totengräber der ohnehin raren Landärzte: Während zweier Jahre hat ein Thaynger Kollege verzweifelt einen Nachfolger gesucht, und nun boykottiert die Helsana drei von diesen vier Landärzten, darunter auch die langersehnte Nachfolgerin. Der einzige Dorfarzt in z. B. Ramsen ist ebenfalls ausgeschlossen. Glauben Sie im Ernst, dass sich unter solchen Umständen ein junger Hausarzt in Zukunft je traut, eine Landpraxis zu übernehmen?

Die Helsana demonstriert in aller Deutlichkeit, womit Patienten und Ärzte rechnen müssen, wenn der Kontrahierungszwang aufgehoben werden sollte und der Entscheid zu Vertragsabschlüssen ohne klare Rahmenbedingungen allein den Krankenkassen überlassen wird.

Allen am Funktionieren unseres Gesundheitswesens interessierten Politikern sollte dieses katastrophale Gebaren die Augen öffnen. Ich fordere Sie auf, endlich für transparente und klare Rahmenbedingungen zu sorgen, die Macht der Kassen zu beschränken und für grundsätzlich paritätisch zusammengesetzte Entscheidungsgremien in allen Belangen zu sorgen!

Wie wäre es denn, wenn wir Ärzte bei diesen alternativen Modellen von unserer Behandlungspflicht ebenfalls befreit würden und zum Beispiel Helsana-Patienten nicht mehr behandeln würden? Das wären gleich lange Spiesse!

Sie werden argumentieren, dass Sie für Vertragsverhandlungen bereit seien. Wir waren dazu bereit und haben uns ernüchtert wieder zurückgezogen. Erstens hält sich unsere Bereitschaft zur Zusammenarbeit aufgrund unserer Erfahrungen mit einem so unzuverlässigen Partner in Grenzen. Zweitens ist Ihre geforderte Beschränkung auf maximal 40\% der Hausärzte auf Ihrer Auswahlliste indiskutabel! Bedenklich sind auch Ihre weiteren Forderungen, ich erwähne nur die verlangte fast unbeschränkte Einflussnahme Ihrer Casemanager in Behandlungen, ohne vertragliche Zusicherung, dass der letzte Entscheid immer noch beim Arzt und nicht bei einem Laien liegen darf! Auch viele weitere Einmi- 
schungs- und Kontrollkriterien übersteigen das tolerierbare Mass. Schuster, bleib bei deinen Leisten: Wir erwarten, dass Sie sich um das Versicherungsgeschäft kümmern und wir Ärzte - und nicht die Krankenkassen - für die Behandlungen zuständig sind! Unter den von Ihnen diktierten obenerwähnten Bedingungen kommt keine $\mathrm{Zu}$ sammenarbeit zustande.

Dass sich die Helsana um gesetzliche und vertragliche Bestimmungen foutiert, rundet das Bild ab. Ich erwähne nur kurz die von der Helsana heftig beworbenen und dennoch nicht legalisierten Auslandbehandlungen (vielleicht wäre auch das Management und die Verwaltung in
Deutschland günstiger?) oder Ihre Versuche, die Patienten mit Briefen für die vertragswidrige $\mathrm{Ab}$ geltung nach dem System des «tiers payant» über das kasseneigene Rechenzentrum zu ködern.

Der Verein für Hausarztmedizin im Kanton Schaffhausen zählt zu den engagierten Netzwerken in der Schweiz. Wir sind - und beweisen dies zusammen mit anderen, vernünftigeren Krankenkassen laufend - zu einer kooperativen, fairen und partnerschaftlichen Zusammenarbeit bereit. Wir wehren uns aber vehement gegen unfaire Machenschaften und einseitige Machtdemonstrationen. Ich fordere Sie zu einer Umkehr von Ihrer eingeschlagenen Strategie auf.

\section{Förderung und Bewertung der Qualität in Ärztenetzwerken mit dem Indikatorenset Quali-med-net ${ }^{\circledR}$}

\author{
Peter Christen, Karl Metzger, \\ Arbeitsgruppe Qualität ${ }^{a}$, \\ med-swiss.net \\ a Arbeitsgruppe Qualität med- \\ swiss.net: Peter Christen (Leiter \\ Arbeitsgruppe, zmed und VHZ), \\ Wolfgang Czerwenka (argomed), \\ Rainer Hurni (zmed und VHZ), \\ Christof Kaech (Biel-Seeland), \\ Karl Metzger (BlueCare), Andreas \\ Schneider (thurcare/VMCT), \\ Hans Beat Stiefel (hawa/hawadoc), \\ Georg von Below (FMH, bis 2005), \\ Axel Buchli (SGAM, bis 2005).
}

Fussnoten auf der nächsten Seite.

Résumé voir page suivante

Korrespondenzen:

Dr. med. Peter Christen

Facharzt Allgemeinmedizin FMH

Limmattalstrasse 177

CH-8049 Zürich

Tel. 0443418600

Fax 0443418626

peterchristen@hin.ch

Karl Metzger

Leiter Managed Care

c/o BlueCare

Pflanzschulstrasse 3

CH-8400 Winterthur

Tel. 0522351935

Fax 0522351920

karl.metzger@bluecare.ch

\begin{abstract}
Einleitung
Von integrierten Versorgungsnetzwerken ${ }^{1}$ wird neben einer besseren Qualität der Versorgung auch ein kostendämpfender Beitrag in den wachsenden Gesundheitskosten erwartet. In Zukunft wird die ökonomische Mitverantwortung der Leistungserbringer im Bereich von ManagedCare-Systemen durch die Vereinbarung sogenannter Budgetverträge an Bedeutung gewinnen. Ob sich auch die Politik für die Ausgestaltung sinnvoller Managed-Care-Anreize im Rahmen der laufenden KVG-Revision einsetzen wird, ist momentan noch offen. ${ }^{2}$ Im Zuge dieser Entwicklung wird die Darstellung der Versorgungsqualität in Ärztenetzwerken eine besondere Herausforderung sein. Die Arbeitsgruppe Qualität des Dachverbandes der Schweizer Ärztenetzwerke med-swiss.net ${ }^{3}$ - hat vor diesem Hintergrund sowie auf Basis von gesammelten Erfahrungen in Ärztenetzwerken Quali-med-net - ein Qualitätsindikatorenset für Ärztenetzwerke - entwickelt. Erste Erfahrungen in der Anwendung dieses Instruments zeigen die Vorteile des flexiblen und modularen Aufbaus, aber auch die Hürden in der Umsetzung, die es zu berücksichtigen gilt.
\end{abstract}

Qualitätsentwicklung in den Netzwerken Ärztenetzwerke engagieren sich - durch die Bündelung kollektiven Wissens - traditionell in der Qualitätsoptimierung und Versorgungssicherheit.
Die umfassende, vernetzte und kostenbewusste Betreuung der Patienten ist ein Hauptmerkmal professioneller Ärztenetzwerke und ein entscheidender Erfolgsfaktor für deren Existenz. Mit der Unterstützung der Netzärzte zur verbindlichen Teilnahme an Qualitätszirkeln, am europäischen Praxisassessment EPA oder an einem Critical Incidence Reporting, haben die Ärztenetzwerke wichtige Impulse für die Qualitätsentwicklung gesetzt.

\section{Das Indikatorenset Quali-med-net ${ }^{\circledR}$}

Das Instrument Quali-med-net fasst den Begriff der Qualitätsentwicklung in Ärztenetzen weiter: Mit diesem Selbstbeurteilungsmodell können eine Vielzahl der Leistungen und Entwicklungsprozesse in einem Ärztenetzwerk dargestellt und Qualitätsförderungsmassnahmen als lernendes System, von der Ärztebasis ausgehend - bottomup -, entlang einem Reifegradmodell umgesetzt werden.

An die Entwicklung des Indikatorensets wurden folgende Ziele geknüpft: Das Instrument soll allen Ärztenetzwerken zugänglich sein, die Möglichkeit zur Weiterentwicklung geben, als Auswahl- und Orientierungshilfe dienen, Anpassungen je nach Qualitätsbedürfnis erlauben und auch die Möglichkeit zum Benchmarking (kollegiale Bestresultate) geben. 
1 Ärztenetze sind Organisationen, die von Leistungserbringern gebildet werden und der Gesundheitsversorgung dienen. Durch verbindliches Zusammenwirken unter sich, mit netzfremden Leistungserbringern und mit den Kostenträgern erbringen sie auf die Bedürfnisse der Patientinnen und Patienten ausgerichtete Gesundheitsleistungen. Dieses Zusammenwirken beruht auf vereinbarten Behandlungsprozessen unternehmerischen Organisationsstrukturen und einer gemeinsamen Betreuungskultur. Sie setzen sich bei hoher Versorgungsqualität einen ökonomischen Umgang mit den vorhandenen Mitteln zum Ziel. Ein weiteres Merkmal der Versorgungsnetze ist der Wille, die Gesundheitsleistungen aus einer Hand zu steuern (in Anlehnung an die Definition des med-swiss.net im Grundlagenpapier «Netzwerke - von der Vision zur Realität» vom 19. Januar 2005)

2 Stand November 2006, Impulse werden von der Ständeratsdebatte im Dezember 2006 erwartet.

3 Dem med-swiss.net sind momen$\tan 38$ Ärztenetzwerke mit über 1500 Netzärzten angeschlossen (siehe www.med-swiss.net)

Tabelle 1

Beispiel eines Indikators.

\section{Indikator}

4.1 Informationen für Patienten, Netzärzte und Partner

\section{Messgrösse \\ Informationsschriften \\ vorhanden: ja/nein}

Standard

Regelmässige, periodische Informationen
Entwicklung/Ausbau Spezifische Informationen je Zielgruppe
Mit dem Indikatorenset soll keine neue Kontrollbürokratie entstehen. Die Abläufe und Prozesse sollen durch die Ärztenetzwerke selbst definiert werden (bottom-up). Die einzelnen Indikatoren sollen hauptsächlich prozess- und ergebnisorientiert sein.

\section{Aufbau des Quali-med-net ${ }^{\circledR}$ \\ Indikatorensets}

Die Indikatoren sind in fünf Qualitätsdimensionen gegliedert:

- Mitgliedschaft, Organisation und Infrastruktur;

- interne und externe Zusammenarbeit;

- Prozessmanagement;

- Kommunikation;

- Outcome.

Für jede Dimension ist eine Anzahl Indikatoren definiert. Als Indikator ist das Instrument zur Leistungsbewertung zu verstehen (im Gegensatz zu Standards bei der Qualitätsbemessung). Das Indikatorenset besteht momentan aus 43 Indikatoren. Je Indikator wurden eine Messgrösse, ein Standard und ein Weiterentwicklungsvorschlag definiert (Tab. 1).

Jeder Indikator wurde in einem Arbeitshandbuch mit ausführenden Erläuterungen zum Nutzen und zur Messung des Indikators ergänzt. Das Handbuch dient den Ärztenetzwerken dabei auch als Orientierungs- und Nachschlagewerk.

\section{Anwendung}

Vor einer Beschäftigung mit Qualitätsindikatoren sollte sich die Leitung eines Ärztenetzes mit dem Profil und dem Entwicklungsstand ihres Ärztenetzes auseinandersetzen. Mit der Erfassung des Ist-Zustandes können je nach regionalen Gegebenheiten und Bedürfnissen jene Indikatoren ausgewählt und priorisiert werden, die die Netzwerkentwicklung zum gegebenen Zeitpunkt am besten unterstützen. Es lohnt sich dabei, die verfolgten Indikatoren mit dem Umfeld - den Partnern des Ärztenetzwerkes - abzustimmen, um die notwendige Akzeptanz für das Umsetzbare zu schaffen. Qualitätsentwicklung ist eine Team- arbeit aller Netzärzte, die von der Netzleitung koordiniert und dokumentiert werden muss. Periodisch soll Rechenschaft darüber abgelegt werden, was erreicht wurde und wo es Abweichungen gibt. Ist der Soll-Zustand erreicht, gilt es, diesen zu bewahren, damit das erreichte Qualitätsniveau auch in Zukunft gehalten werden kann.

\section{Promotion et évaluation de la qualité des réseaux de médecins grâce aux groupes d'indicateurs Quali-med-net ${ }^{\circledR}$}

On attend des réseaux de fourniture de soins une meilleure qualité de l'assistance et un apport à la réduction des dépenses dans la spirale ascendante des coûts de la santé. La coresponsabilité économique des prestataires de soins dans les systèmes de managed care devait prendre de l'importance par la conclusion de conventions dites budgétaires. La question demeure ouverte de savoir si les politiques développeront eux aussi des incitations raisonnables pour favoriser la gestion des soins dans le cadre de la révision continue de la LAMal. Dans ce contexte, la représentation de la qualité des soins au sein des réseaux de médecins représente un défi particulier. Le groupe de travail qualité de l'Organisation faîtière des réseaux médicaux suisses (Quali-med-net) a développé sur cette base, et en s'inspirant des expériences faites, un groupe d'indicateurs pour les réseaux de médecins. Les premiers tests de cet instrument montrent les avantages d'une structure souple et modulaire, mais aussi les obstacles à son application, dont il faudra tenir compte. 


\section{Umsetzung}

Im Herbst 2005 wurde allen Ärztenetzwerken von med-swiss.net das Arbeitshandbuch zur Vernehmlassung und ersten Umsetzung zur Verfügung gestellt. Seither wurde insbesondere in den Ärztenetzwerken der Arbeitsgruppenmitglieder der geschilderte Evaluationsprozess eingeleitet. Es hat sich herausgestellt, dass für einen Jahreszyklus die Auswahl und Bearbeitung von einer nur sehr beschränkten Anzahl (ein bis fünf) Indikatoren sinnvoll ist, da das Einleiten von Entwicklungs- und Umsetzungsprozessen mit einem nicht $\mathrm{zu}$ unterschätzenden Aufwand verbunden ist. Dabei kann es sinnvoll sein, zusammen mit den Partnern (z. B. den Versicherern) solche Projekte zu definieren und eine Finanzierungslösung zu vereinbaren. Die bisher gewonnenen Erfahrungen zeigen, dass Quali-med-net ein taugliches und motivierendes Instrument für Ärztenetzwerke ist, Qualitätslücken zu identifizieren und Verbesserungen einzuleiten. Die Ärztenetzwerke haben damit ein Instrument, das nach ihren Vorstellungen entwickelt wurde und angewendet werden kann.

\section{Ausblick}

Eine weitere Verbreitung von Quali-med-net ist wünschenswert, um die Leistungsqualität von Ärztenetzen kontinuierlich zu verbessern und der Netzwerkqualität auch ein Profil zu geben. Von seiten der Versicherer besteht ein grosses Interesse an einem transparenten Qualitätsprofil ihrer Partner. Dieser Wunsch ist zwar verständlich, weckt aber gleichzeitig auch die Versuchung, unnötige Kontroll- und Sanktionsinstrumente einzuführen.

Die Philosophie von Quali-med-net ist aber eine andere: Die Netzwerkqualität, wie sie Qualimed-net formuliert, geht weit über die vom Gesetzgeber geforderte Qualitätssicherung hinaus und ist eine freiwillige Leistung hin zu einer optimalen Versorgungsqualität. Nur motivierte Qualitätsentwicklung führt auch zu echter Qualitätsverbesserung. In Analogie zum Ökolabel «Knospe» könnte ein Netzwerkqualitätslabel diese Entwicklung unterstützen. Die Nürnberger Praxisnetzstudie 2006 [1] hat dazu ein Stufenmodell vom «Einsteiger» bis zum «Netzprofi» vorgestellt. Für qualifizierte Ärztenetzwerke ergäben sich mit einem Qualitätslabel weitere Vorteile: die Unterstützung für eine Netzidentität («Corporate identity») und die gestärkte Positionierung im Gesundheitsmarkt, beispielsweise in den Managed-Care-Verträgen mit den Versicherungen. Die Zertifizierung eines ganzen Ärztenetzwerkes, so wie dies die EQUAM-Stiftung anbietet, ist eine Option, die Ärztenetzwerke bei Bedarf später als ein nächstes Ziel anstreben können.

Ergänzende Informationen zu Quali-med-net sind erhältlich bei med-swiss.net oder bei den Autoren.

\section{Literatur}

1 Schicker G et al. Praxisnetzstudie 200. Arbeitspapier 1/2006. Universität Erlangen-Nürnberg; 2006. 